\title{
Editorial: Thermal Hydraulics and Safety Analysis in Generation-IV Reactors
}

\author{
Dalin Zhang ${ }^{1 *}$, Jian Deng ${ }^{2}$, Zhanjie $X u^{3}$ and Michitsugu Mori ${ }^{4}$ \\ ${ }^{1}$ School of Nuclear Science and Technology, Xi'an Jiaotong University, Xi'an, China, ${ }^{2}$ Science and Technology on Reactor \\ System Design Technology Laboratory, Nuclear Power Institute of China, Chengdu, China, ${ }^{3}$ Institute for Nuclear and Energy \\ Technologies, Karlsruhe Institute of Technology, Karlsruhe, Germany, ${ }^{4}$ Division of Energy and Environmental Systems, \\ Graduate School of Engineering, Hokkaido University, Sapporo, Japan
}

Keywords: thermal-hydraulic, reactor safery, Gen-IV reactors, floating reactor, computational fluid dynamics modeling

\section{Editorial on the Research Topic}

\section{Thermal Hydraulics and Safety Analysis in Generation-IV Reactors}

For the sustainable development of nuclear energy, the US Department of Energy (DOE) established the Generation IV International Forum (GIF) in collaboration with 12 other countries to develop advanced Generation-IV reactors. Six types of reactors were chosen as candidates, and the thermal-hydraulic and safety analysis of Gen-IV reactors has attracted worldwide attention.

With more and more research focused on the Gen-IV reactors, a large number of valuable results and publications have emerged in recent years. The Research Topic "Thermal Hydraulics and Safety Analysis in Generation-IV Reactors" aims to publish the most advanced and timely research results related to thermal-hydraulic and safety analysis of Gen-IV reactors around the world. The issue has been organized by Dalin Zhang from Xi'an Jiaotong University, Jian Deng from Nuclear Power Institute of China, Zhanjie Xu from Karlsruhe Institute of Technology, and Michitsugu Mori from Hokkaido University. The articles include experimental and numerical research results on Gen-IV reactors and other innovative reactors, and some special phenomena and mechanisms are also included.

Wang, Chen, Wen et al. reviewed the research development of the lead-bismuth research reactors in China in the article "A Mini Review of Research Progress of Nuclear Physics and Thermal Hydraulic Characteristics of Lead-Bismuth Research Reactor in China." The safety analysis of the lead-bismuth research reactors is greatly addressed by Chinese scholars. Although the research on the lead-bismuth reactors began late in China, Chinese scholars have made great progress.

To improve the efficiency and accuracy of the numerical simulation of the fuel assemblies with wire spacers, which is widely used in the Gen-IV reactors, Wang X. A. et al. developed a hexahedron meshing strategy based on Radial Basis Function (RBF) theory in the article "Generating Hexahedral Mesh for Wire-wrapped Fuel Assembly with RBF Mesh Deformation Method." The strategy is validated by two series of experimental results, and the models will be beneficial for the modeling of the wire-wrapped fuel assemblies in a real nuclear reactor core with a large number of fuel pins.

Jie summarized the recent results of the thermal-hydraulic research on the floating nuclear power plants in China in the article "Research Progress of Reactor Thermal-Hydraulic Characteristics Under Ocean Conditions in China." The authors emphasized the importance of flow and heat transfer, bubble behavior, flow instability, and critical heat flux under ocean conditions.

Wang, Chen, Ke et al. conducted experimental research on the flow characteristics of the 
liquid film on the wall surface of the corrugated plate dryer in the article "Analysis of the Flow Characteristics of the Liquid Film on the Wall Surface of the Corrugated Plate Dryer Based on PSD Method: A Short Communication." The plane laser induced fluorescence (PLIF) method was adopted to measure liquid film thickness and the power spectral density (PSD) method was used to study the non-linear characteristics of liquid film thickness. The experimental results showed that the relationship between film thickness and the Reynolds number, as well as the deviation from Nusselt and Karapantsios estimation.

Li et al. constructed a transient test device to study the transient characteristics of a nuclear reactor coolant pump in coast down process under power failure condition in the article "An Experimental Study on Transient Characteristics of a Nuclear Reactor Coolant Pump in Coast-Down Process Under Power Failure Condition." The experimental results are of great importance in controlling the stability and reliability of the nuclear reactor coolant pump in the coast-down process.

Long et al. used the transient fluid-solid coupling to analyze the pump shaft stability of the TDM $35-140 \times 13$ multistage centrifugal pump during the closed-valve start-up process in the article "Research on the Pump Shaft Stability Analysis of Multistage Centrifugal Pump During Closed-Valve Start-Up Process."
In general, thanks to the efforts of the reviewers and editors, a total of six articles from all over the world have been collected, covering both review articles and research articles, experimental research and numerical research, and Gen-IV reactors and other innovative reactors. We would like to show our gratitude to all the reviewers, authors, and Frontiers staff in the Energy Research editorial office for their help and support.

\section{AUTHOR CONTRIBUTIONS}

All authors listed have made a substantial, direct and intellectual contribution to the work, and approved it for publication.

Conflict of Interest: JD was employed by Nuclear Power Institute of China.

The remaining authors declare that the research was conducted in the absence of any commercial or financial relationships that could be construed as a potential conflict of interest.

Copyright (c) 2021 Zhang, Deng, Xu and Mori. This is an open-access article distributed under the terms of the Creative Commons Attribution License (CC BY). The use, distribution or reproduction in other forums is permitted, provided the original author(s) and the copyright owner(s) are credited and that the original publication in this journal is cited, in accordance with accepted academic practice. No use, distribution or reproduction is permitted which does not comply with these terms. 